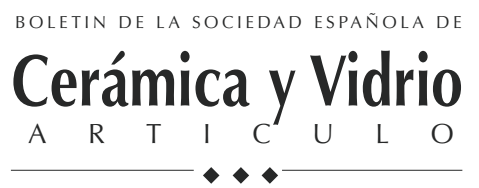

\title{
Obtención de $\mathrm{Si}_{3} \mathrm{~N}_{4}$ mediante SHS
}

\author{
I.G. CANO, M.A. RODRÍGUEZ \\ Instituto de Cerámica y Vídrio (CSIC), Campus de Cantoblanco, Madrid, Spain
}

\begin{abstract}
En el presente trabajo se presentan los resultados obtenidos en el estudio de las reacciones de Síntesis Autopropagada a Alta Temperatura (Self-propagating High-temperature Synthesis, SHS) de Nitruro de Silicio. La síntesis autopropagada a alta temperatura consiste básicamente en la generación de reacciones altamente exotérmicas capaces de automantenerse. Se puede considerar como principal ventaja del método el ahorro energético que supone. La síntesis se realiza sobre una mezcla inicial de silicio metálico sobre la cual se realizan adiciones de diluyente y otros aditivos (sales amónicas) que afectan al desarrollo de la reacción. Se ha estudiado la influencia que en este sistema pueden tener las proporciones de las distintas incorporaciones en la mezcla, tanto en el material resultante como en las condiciones de reacción. Igualmente se ha estudiado la posibilidad de utilización de nuevos aditivos que puedan minimizar el impacto medio ambiental. Se presentan los estudios microestructurales del material obtenido, la identificación cristalográfica de las fases presentes así como los comportamientos de los parámetros que definen la propia reacción. Con la información obtenida se propone el mecanismo predominante de la síntesis del Nitruro de Silicio mediante SHS.
\end{abstract}

Palabras clave: Síntesis, Nitruro de Silicio, SHS

\section{Synthesis of $\mathrm{Si}_{3} \mathrm{~N}_{4}$}

In the present paper results obtained during synthesis of Silicon Nitride by Self-propagating High-temperature Synthesis (SHS) are shown. Self-propagating High-temperature Synthesis is based on the high enthalpy of certain reactions able to be self-sustained. One of the most important advantages of the method is its very low energy consumption. The synthesis is carried out with a mixture of silicon powder with some additions of diluents and other synthesis aids (ammonium salts). The influence of the mixture composition on reaction parameters and characteristics of the obtained products has been studied. The use of new synthesis aids has been also studied in order to decrease the environmental impact of the process. Microstructural study, XRD and reaction parameters are shown. Finally a mechanism of Silicon Nitride synthesis is proposed.

Key words: Synthesis, Silicon Nitride, SHS

\section{INTRODUCCIÓN}

La síntesis autopropagada a alta temperatura (conocida como SHS $)^{[1,2]}$ consiste básicamente en la generación de reacciones altamente exotérmicas capaces de propagarse a traves de la mezcla inicial, tranformandola en los compuestos deseados, sin aporte externo de energía. Se puede considerar como principal ventaja del método el ahorro energético que supone, principalmente si se compara con los métodos utilizados convencionalmente.

Como es bien conocido la síntesis de nitruro de silicio $\left(\mathrm{Si}_{3} \mathrm{~N}_{4}\right)$ se puede realizar por distintas vías, una de las cuales es a través de la reacción de silicio metálico en atmósfera de nitrógeno la cual bajo las condiciones adecuadas, es una reacción autopropagada ${ }^{[3}{ }^{4]}$. La primera restricción que se ha observado en esta reacción SHS es debida a que la temperatura de fusion del silicio es inferior a la temperatura del frente de combustión y por lo tanto, se hace necesario diluir el silicio inicial para evitar la coalescencia de éste y la disminución de su actividad química. Igualmente se hace preciso mantener una elevada presión de nitrógeno debido a la temperatura de des- composición del $\mathrm{Si}_{3} \mathrm{~N}_{4}\left(\sim 1400^{\circ} \mathrm{C}\right)$. Es decir, para que la nitruración de silicio metálico tenga lugar de forma autosostenida es necesario incorporar aditivos de síntesis así como trabajar a altas presiones de nitrógeno.

La dilución de la mezcla se realiza mediante la incorporación de $\mathrm{Si}_{3} \mathrm{~N}_{4}$-diluyente. La adición de pequeñas cantidades de sales amónicas ${ }^{[5]}\left(\mathrm{NH}_{4} \mathrm{Cl}\right.$ y $\left.\mathrm{NH}_{4} \mathrm{~F}\right)$ pueden mejorar el proceso. Cálculos termodinámicos realizados muestran que la adición de estas pequeñas cantidades de especies de amoniaco favorecen significativamente la volatilización del silicio y, se ha comprobado experimentalmente, se producen considerables aumentos en el contenido de $\alpha-\mathrm{Si}_{3} \mathrm{~N}_{4}$ incluso llegando a valores de más del 95\% ${ }^{[6]}$.Sin embargo desde un punto de vista tecnológico los gases originados, en estos casos, producen corrosión tanto en el interior como en el exterior del reactor, siendo además necesario tratar los gases residuales para evitar el deterioro medioambiental.

En concreto en este trabajo se ha tratado en primer lugar, la posibilidad de sustituir las adiciones de $\mathrm{NH}_{4} \mathrm{Cl}$ y/o $\mathrm{NH}_{4} \mathrm{~F}$ 
por otras sales menos agresivas y comparar los productos resultantes con aquellos obtenidos con el empleo de las sales amónicas normalmente utilizadas.

Por otra parte tambien se ha abordado el estudio de parámetros específicos de la reacción como la presión inicial de nitrógeno y la porosidad inicial de la mezcla, tratando de evaluar los límites para que la síntesis sea autosostenida, así como el efecto que las variaciones de estos parámetros causan en las características de los productos obtenidos.

\section{PARTE EXPERIMENTAL}

Las materias primas utilizadas han sido: silicio metálico (pureza > 99\%, $\mathrm{d}_{50}=4 \mu \mathrm{m}$ ); $\mathrm{Si}_{3} \mathrm{~N}_{4}$-diluyente obtenido en el laboratorio por SHS $\left(16,7 \% \alpha-\mathrm{Si}_{3} \mathrm{~N}_{4}, 77,2 \% \beta-\mathrm{Si}_{3} \mathrm{~N}_{4}\right.$ y $6,1 \%$ silicio no reaccionado) y como sales amónicas, las indicadas en la tabla I.

La síntesis se ha realizado en un reactor de alta presión $\left(\mathrm{P}_{\max }=25 \mathrm{MPa}\right)$ de 2 litros de capacidad, en cuyo interior se ha depositado la mezcla inicial sobre una bandeja de grafito. La reacción se ha iniciado en uno de los extremos de la mezcla utilizando una resistencia de $\mathrm{W}$ inmersa en una pequeña cantidad de mezcla de Ti:C (1:1) y, por la cual se ha hecho pasar un pulso eléctrico ( 24 voltios, $5 \mathrm{~s}$.).

Las composiciones iniciales de la mezcla de silicio y aditivos ha variado en función del parámetro estudiado, así en el caso del análisis de sales amónicas se han considerado composiciones similares con un $5 \% \mathrm{~mol}$ de cada una de las distintas sales seleccionadas. Sin embargo, en el caso del estudio del efecto de la presión inicial de nitrógeno se han seleccionado dos formulaciones iniciales bien distintas desde el punto de vista del producto obtenido, es decir, se ha ensayado una formulación con la cual se obtienen altos contenidos en la fase $\alpha-\mathrm{Si}_{3} \mathrm{~N}_{4}$ y otra cuyo producto es rico en la fase $\beta-\mathrm{Si}_{3} \mathrm{~N}_{4}$.

Las fases presentes en el $\mathrm{Si}_{3} \mathrm{~N}_{4}$ final se han determinado mediante difracción de rayosX (Siemens D-5000). El contenido de las distintas fases de $\mathrm{Si}_{3} \mathrm{~N}_{4}$ se han calculado de acuerdo con el metodo propuesto por Gazzara y Messier ${ }^{[7]}$ y el contenido de silicio no reaccionado se ha cuantificado utilizando el método de adiciones sucesivas ${ }^{[8]}$. Las velocidades del frente de reacción han sido evaluadas mediante la colocación en el interior de la mezcla inicial de termopares (W/5\% Re-W/ 20\% $\mathrm{Re}$ ) a distancias conocidas. Las morfologías de los productos han sido observadas por microscopía electrónica de barrido (C. Zeiss, DSM-950).

\section{RESULTADOS Y DISCUSION}

\subsection{Utilización de sales amónicas:}

Como se ha comentado en la parte experimental, las experiencias realizadas han sido identicas en cuanto a la formulación inicial con la salvedad del tipo de sal no utilizando dilución. En todos los casos los compactos resultantes han tenido un aspecto externo similar observandose un considerable esponjamiento debido fundamentalmente a la descomposición de las sales amónicas. Un ejemplo de las fases encontradas se muestra en el difractograma de la figura 1 donde se pueden apreciar las posiciones de los picos característicos de éstas, pudiéndose apreciar la aparición de otras fases, como carburo de silicio $(\mathrm{SiC})$ y oxinitruros de silicio $\left(\mathrm{Si}_{2} \mathrm{ON}_{2}\right)$ si se compara
TABLA I: SALES AMÓNICAS UTILIZADAS Y SUS CARACTERÍSTICAS

\begin{tabular}{|l|l|l|l|}
\hline Sal amónica & Fórmula química & Pureza & Procedencia \\
\hline Cloruro amónico & $\mathrm{NH}_{4} \mathrm{Cl}$ & $>98 \%$ & MERCK \\
\hline Carbamato amónico & $\mathrm{NH}_{4} \mathrm{CO}_{2} \mathrm{NH}_{2}$ & $>99,5 \%$ & MERCK \\
\hline Oxalato amónico & $\left(\mathrm{NH}_{4}\right)_{2} \mathrm{C}_{2} \mathrm{O}_{4} \cdot \mathrm{H}_{2} \mathrm{O}$ & $>99,5 \%$ & MERCK \\
\hline Acetato amónico & $\mathrm{CH}_{3} \mathrm{COONH}_{4}$ & $>98 \%$ & MERCK \\
\hline Carbonato amónico & $\mathrm{NH}_{3} \mathrm{CO}_{3} \mathrm{H}+\mathrm{NH}_{2} \mathrm{COONH}_{4}$ & $>99 \%$ & PANREAC \\
\hline
\end{tabular}

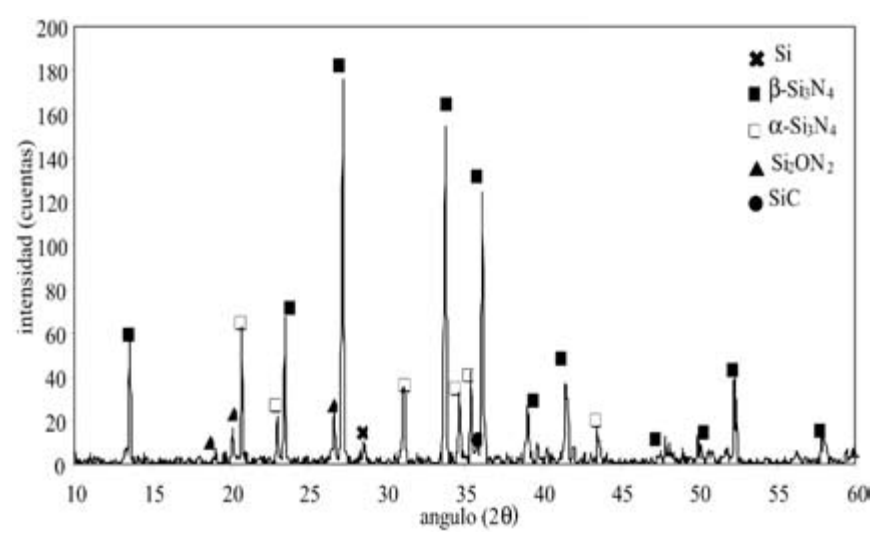

Figura 1: DRX del $\mathrm{Si}_{3} \mathrm{~N}_{4}$ obtenido con la utilización de oxalato amónico como aditivo de síntesis.

con las fases de los productos obtenidos utilizando $\mathrm{NH}_{4} \mathrm{Cl}$ únicamente, además de $\mathrm{Si} \mathrm{y} \mathrm{Si}_{3} \mathrm{~N}_{4}{ }^{[9]}$. La formación de estos compuestos se debe a la descomposición de las sales orgánicas con el . Así, la aparición de estas nuevas fases se debe a la reacción del silicio con los productos de descomposición de los radicales orgánicos. Otra diferencia considerable de los productos ha sido la morfología del $\mathrm{Si}_{3} \mathrm{~N}_{4}$ obtenidos. Mientras que con adiciones de $\mathrm{NH}_{4} \mathrm{Cl}^{[9]}$ se obtienen productos con morfología diversa pero formadas en general por fibras bien definidas, con estas nuevas sales las morfologías son poco uniformes y en ocasiones parecen fuertemente deformadas (figura 2).

En líneas generales los productos finales tienen altos grados de conversión con contenidos de silicio residual menores al $6 \%$ en peso, lo que supone que realmente estas sales mejoran la nitruración con respecto a la síntesis utilizando únicamente Si y $\mathrm{Si}_{3} \mathrm{~N}_{4}(9)$. Solo en el caso de la utilización de carbonato amónico los niveles de silicio no reaccionado han superado el $10 \%$ en peso. En cuanto a los contenidos relativos de fases de nitruro de silicio, se ha observado que la fase $\alpha-\mathrm{Si}_{3} \mathrm{~N}_{4}$ generada está entre un $20 \%$ y $30 \%$ como muestra la figura 3 y, es el carbonato amónico la sal con la que se obtienen contenidos mayores de esta fase (del orden del 50\% en peso). Este hecho indica que es con este aditivo cuando más se rebaja la temperatura del frente, no logrando evitar, no obstante, la coalescencia de parte del silicio metálico, siendo de hecho como ya se ha comentado, el caso donde se han encontrado los niveles mas bajos de reacción. 

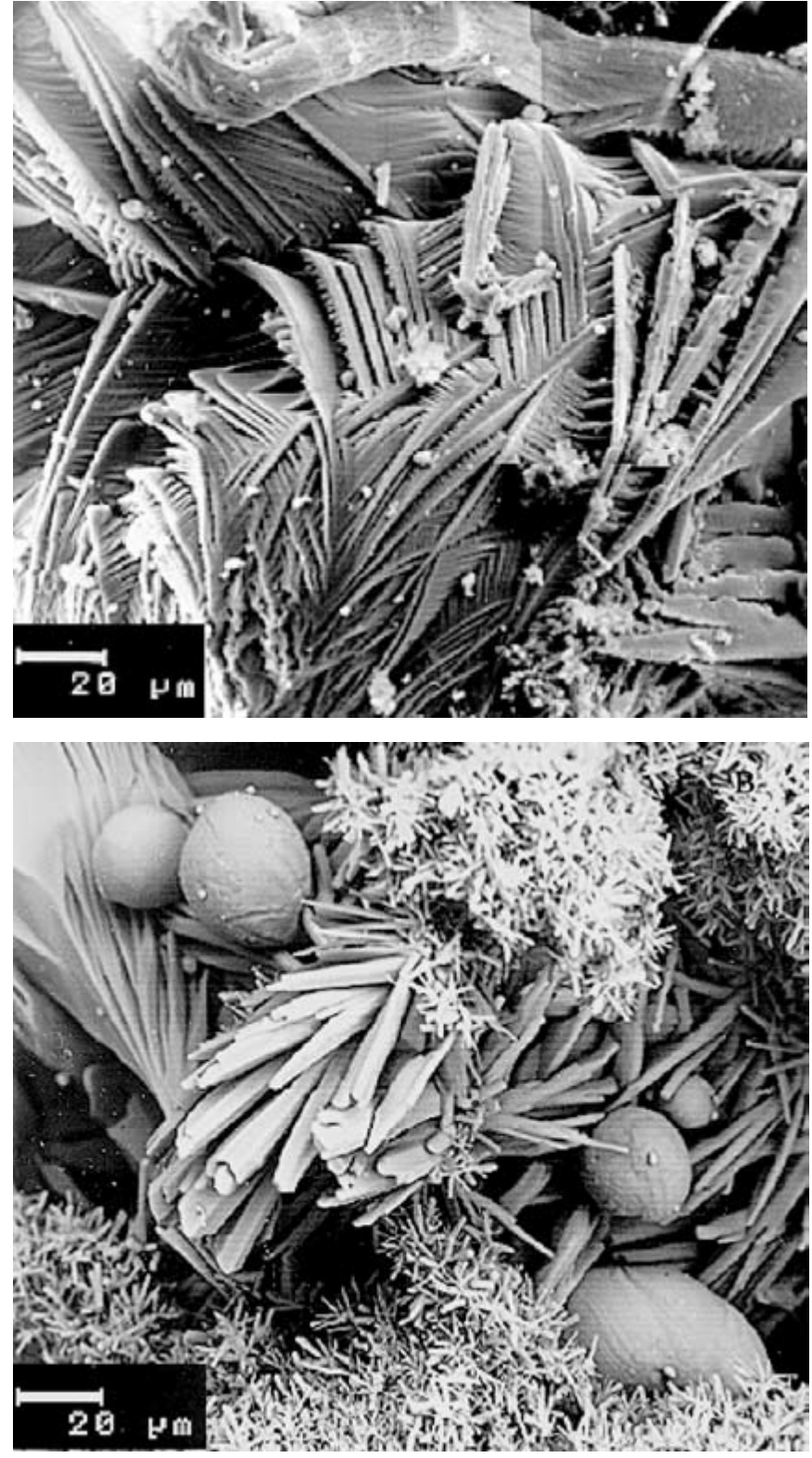

Figura 2 : Micrografía de morfologías correspondientes a la adición de carbonato amónico: A) Distribución en forma de espiga; B) Distribución heterogénea

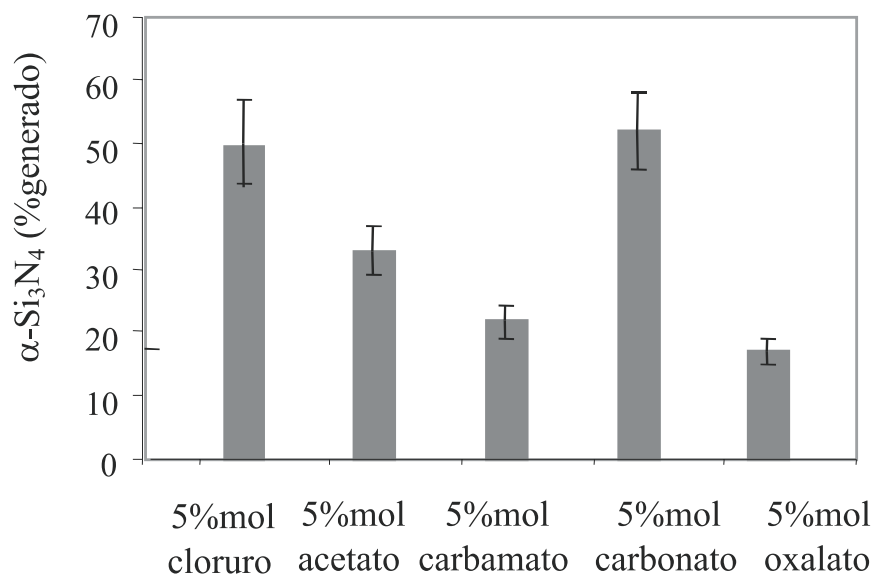

Figura 3: Contenido de fase $\alpha-\mathrm{Si}_{3} \mathrm{~N}_{4}$ generada utilizando distintas sales como aditivos de síntesis
3.2 Limites de la reacción: Otros parámetros de la reacción

\subsubsection{PRESIÓN INICIAL DE NITRÓGENO}

El nitrógeno y el silicio son los componentes reactivos de la reacción, es por ello, que conocer los valores límites para los cuales la reacción SHS tiene o no lugar, es importante. Como se ha descrito en la parte experimental, se han seleccionado dos formulaciones iniciales bien diferentes en cuanto al producto obtenido, para evitar la superposición de efectos con la presión. Como valores mínimos de presión, para los cuales la síntesis era autosostenida, se han obtenido $4 \mathrm{MPa}$ y $8 \mathrm{MPa}$ para formulaciones propicias a la obtención de $\beta-\mathrm{Si}_{3} \mathrm{~N}_{4}(5 \%$ mol Si $\left.\mathrm{N}_{4}, 1 \% \mathrm{~mol} \mathrm{NH} \mathrm{Cl}_{4}\right)$ y de $\alpha-\mathrm{Si}_{3} \mathrm{~N}_{4}\left(2 \% \mathrm{~mol} \mathrm{Si}_{3} \mathrm{~N}_{4}, 2 \% \mathrm{~mol}\right.$ $\mathrm{NH}_{4} \mathrm{Cl}, 3 \% \mathrm{~mol} \mathrm{NH}_{4} \mathrm{~F}$ ), respectivamente.

Con ambas formulaciones se ha observado, como era prevesible, que el contenido de silicio no reaccionado ha disminuido bruscamente con el aumento de presión llegando a valores de conversión del 90\% en el caso de presiones máximas. Lo cual parece lógico si se piensa que al aumentar la presión del nitrógeno aumenta la velocidad de reacción.

La velocidad del frente de combustion ha aumentado con la presión como se puede observar en la figura 4 que, igualmente, muestra el considerable aumento experimentado por el contenido final de fase $\beta-\mathrm{Si}_{3} \mathrm{~N}_{4}$. Este hecho se debe al aumento de la velocidad de generación de energía calorífica, y por lo tanto al aumento de la temperatura de los productos de reacción. Esto provoca la transformación $\alpha \rightarrow \beta$ del $\mathrm{Si}_{3} \mathrm{~N}_{4}$ en el producto obtenido.

\subsubsection{POROSIDAD DE LA MEZCLA INICIAL:}

Las porosidades estudiadas han sido de 80, 60 y $40 \%$, siendo en este último caso imposible conseguir que la reacción tenga lugar de forma autosostenida. En este caso la formulación empleada ha sido $44 \% \mathrm{Si} \mathrm{y} 56 \% \mathrm{Si}_{3} \mathrm{~N}_{4}$. Es cierto que existen referencias ${ }^{[10]}$ donde se habla de estas condiciones como posibles pero quizá no se hayan empleado características o condiciones de reacción similares (por ejemplo, en cuanto a materias primas se refiere). Indicar así mismo que con porosidades del $60 \%$ la reacción no tiene lugar, de forma autosostenida, para diluciones superiores al $40 \%$.

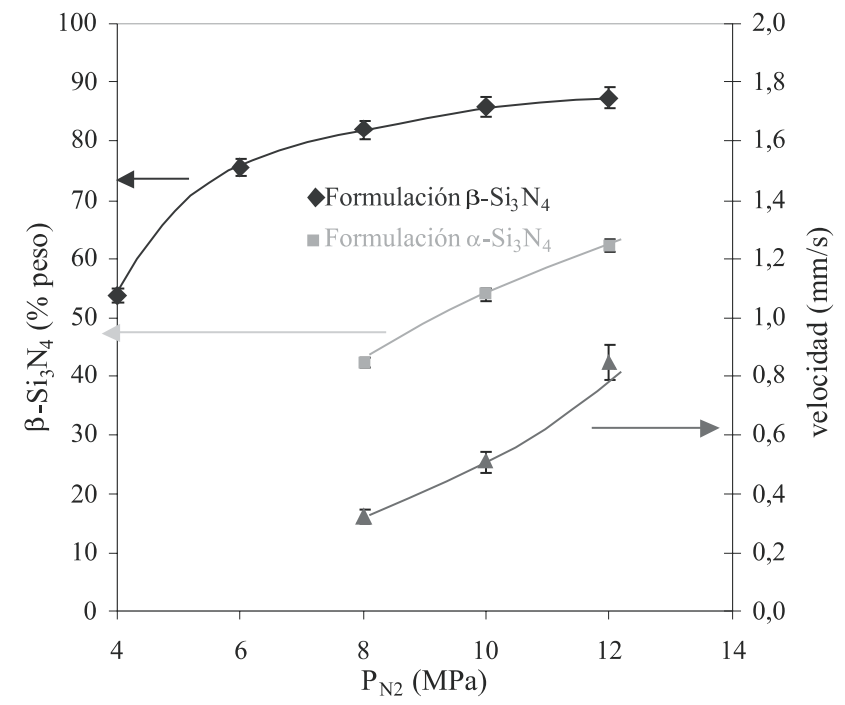

Figura 4: Evolución de la velocidad del frente de combustión y del contenido de $\beta-\mathrm{Si}_{3} \mathrm{~N}_{4}$ en función de la presión inicial de nitrógeno 
Es importante notar que en función de la porosidad se han observado distintos comportamientos según la composición inicial. En el caso de una porosidad del $60 \%$ se ha observado un aumento de la velocidad del frente de reacción con el aumento del contenido de $\mathrm{Si}_{3} \mathrm{~N}_{4}$-diluyente, mientras que para un $80 \%$ de porosidad este comportamiento está claramente dividido en dos zonas como se puede observar en la figura 5. Cuando la dilución es baja $(<40 \%)$ el comportamiento de la velocidad coincide con el de porosidad $60 \%$, produciendose a medida que el diluyente aumenta un aumento de la superficie efectiva de reacción debida a la menor tendencia a la coalescencia de las particulas de silicio. Sin embargo para diluciones $>40 \%$, el aumento de la dilución ha provocado una disminucion de la velocidad del frente ocasionada por la disminucion de la masa reactiva. Asi mismo, es significativo apreciar que la disminución de la porosidad hace más restrictiva la reacción SHS y posiblemente de ahí la diferencia de comportamientos.

\section{CONCLUSIONES}

1.- La sustitución de las sales amónicas $\left(\mathrm{NH}_{4} \mathrm{Cl}, \mathrm{NH}_{4} \mathrm{~F}\right)$ utilizadas habitulamente como aditivos en la mezcla inicial por otras sales derivadas de compuestos orgánicos (acetato, oxalato, carbamato y carbonato amónicos) aporta mejoras sustanciales desde el punto de vista medioambiental, eliminando la emisión de gases corrosivos. Esta sustitución es interesante a nivel industrial aunque ocasiona la aparición en los productos finales de pequeñas cantidades de oxinitruros y/o carburo de silicio que pueden influir en las propiedades de comportamiento del material.

2.- Variaciones de porosidad en la mezcla inicial afectan al comportamiento de la reacción y por tanto, a las características del producto. Para porosidades bajas la reacción es más restrictiva, se observa una velocidad del frente de combustión creciente con la dilución de la mezcla, sin embargo para porosidades del $80 \%$ se han observado dos efectos contrapuestos que originan la aparición de un máximo en la curva de comportamiento dependiendo de cual de estos efectos es el predominante.

3.- El aumento de la presión inicial de nitrógeno provoca aumentos de la velocidad de combustión y es responsable de un significativo aumento en el contenido de la fase $\beta-\mathrm{Si}_{3} \mathrm{~N}_{4}$ generada durante la reacción de síntesis.

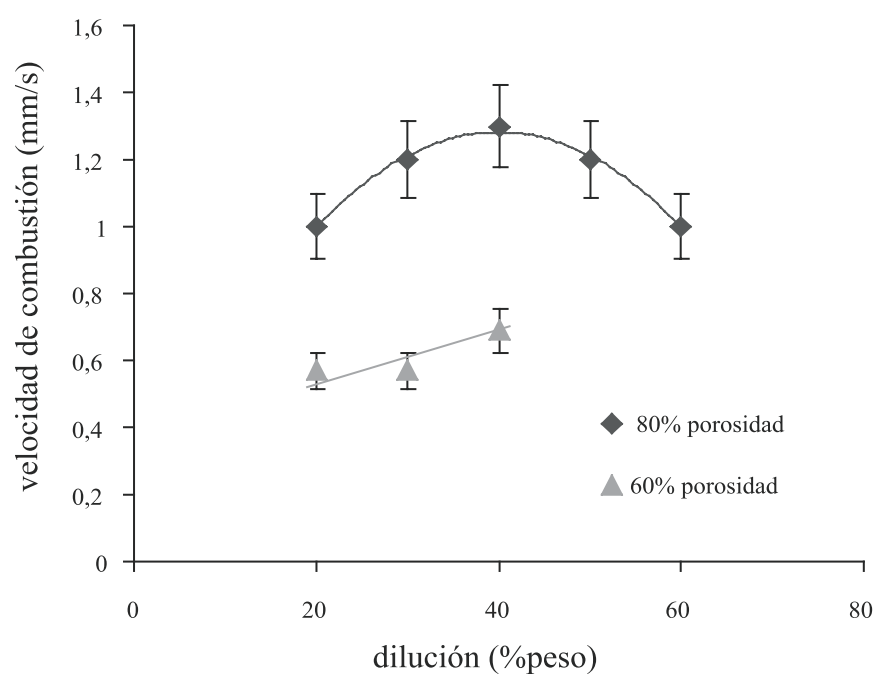

Figura 5: Evolución de la velocidad del frente de combustión en función de la dilución para porosidad de $80 \%$ y $60 \%$

\section{REFERENCIAS}

1. A. G. Merzhanov and I. P. Borovinskaya, "A New Class of Combustion Process", Combust. Sci. Technol. 10, 195-201 (1975).

2. J. Bermudo, M.I. Osendi. "Reacciones autopropagadas a alta temperatura: Fundamentos y aplicaciones". Bol. Soc. Esp. Ceram. Vidr. 35, 7-14 (1996).

3. K. Hirao, Y. Miyamoto, and M. Koizumi. "Synthesis of Silicon Nitride by a Combustion Reaction under High Nitrogen Pressure". J. Am. Ceram. Soc. , 4, 60-62, (1986).

4. Wei-Chang Lee and Shyan- Lung Chung. "Combustion synthesis of $\mathrm{Si}_{3} \mathrm{~N}_{4}$ powder".J. Mater. Res., 12, 805-811, (1997).

5. Yong-Ge Cao, Chang-Chun Ge, Zhang-Jian Zhou, and Jiang-Tao Li. " Combustion synthesis of $\alpha-\mathrm{Si}_{3} \mathrm{~N}_{4}$ whiskers". J. Mater. Res., 14, 876-880 (1999).

6. A.G. Merzhanov, I.P. Borovinskaya, L.S. Popov, N.S. Makhonin, L.V. Kustova, "Method of Obtaining Silicon Nitride with High $\alpha$ Phase Content", WO Patent No 89/11447, (1989)

7. C. P. Gazzara and D. R. Messier. “Determination of Phase Content of $\mathrm{Si}_{3} \mathrm{~N}_{4}$ by X-Ray Diffraction Analysis”. Ceram. Bull., 56, 777-780, (1977).

8. L.E. Copeland and R.H. Bragg. "Quantitative X-Ray Diffraction Analysis", Analitycal Chemistry, 25, 196-201 (1958).

9. I.G. Cano, S.P. Baelo, M.A. Rodríguez, S. de Aza. “ Self-propagating Hightemperature Synthesis of $\mathrm{Si}_{3} \mathrm{Ni}_{4}$ : Role of ammonium salt addition", J. Eur. Ceram. Soc. 21, 291-295 (2001).

10. A.S. Mukasyan, V.M. Martinenko, A.G. Merzhanov, I.P. Borovinskaya, M.Yu. Blinov. "Mechanism and Principles of Silicon Combustion in Nitrogen", Combust. Explos. Shock Waves (Eng. Transl.) 22, 534-554 (1986). 